\title{
A Theory of Diurnal Magnetic Variations in Equatorial Regions and Conductivity of the Ionosphere $E$ Region
}

\author{
By Motokazu HIRONO
}

Geophysical Institute, Kyoto University

\begin{abstract}
In two previous papers, I suggested that the electrical conductivity in $\mathrm{E}-\mathrm{W}$ direction of the $E$ region in a narrow belt along the magnetic equator is much greater than in the other latitudes, on account of a polarization field produced by the Hall Current, provided that $\lambda$ is much less than 10. And that this high conductivity will cause enhanced diurnal magnetic variations near the magnetic equator.

In this paper, I discuss this theory further in detail. A dynamo theory for the $E$ region with anisotropic conductivity, which is linked by highly conductive lines of magnetic force to the $F$ region, is examined. A case that the $E$ layer only makes a tidal oscillation, which produces observed lunar diurnal current system, is calculated. It is found that, in middle latitudes, the induced polarization field in $\mathrm{E}-\mathrm{W}$ direction by the Hall Current is greater than that by the direct current, and the vertical drift of the $F 2$ layer by this polarization field seems to be adequate for the observed $F^{2}$ lunar variation. According to the present theory, conductivity of the $E$ region at the magnetic equator decreases with increase of the main magnetic field, and the distribution of range of magnetic variations along the magnetic equator reported by Egedal, seems to support this relation.
\end{abstract}

\section{Introduction}

In the course of statistical researches of Sq field of terrestrial magnetism in middle and low latitudes, it is noticed that the range of diurnal magnetic variations at Huancayo near the magnetic equator (Dip $2 .{ }^{\circ} 3 \mathrm{~N}$ ) is much greater than at other stations. Whether there are other such places than Huancayo, is a matter of great interest, For the purpose
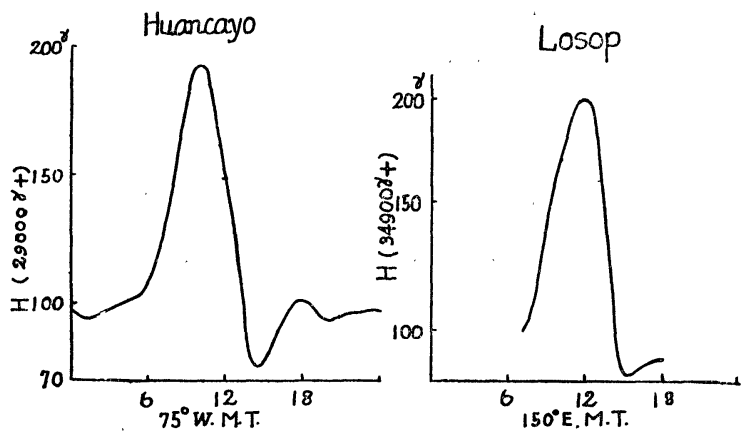

Fig. 1 Diurnal Variation of Magnetic Horizontal Intensity. Mean Values : Losop Feb. 12-15, Huancayo Feb. 11-14 (1934). (After S. Hayami and H, Higashinaka) 
of studying solar eclipse of Feb. 14, 1934, Prof. S. Hayami and H. Higashinaka [1] made magnetic observations at Losop Island $\left(6^{\circ} 53^{\prime} \mathrm{N}, 152^{\circ} 44^{\prime} \mathrm{E}\right.$, Dip $\left.1^{\circ} 45^{\prime} \mathrm{N}\right)$ from Feb. 12 to Feb. 15, which were reported by Prof. M. Hasegawa [3]. In Figure 1 mean $H$ variations are reproduced for Losop (Feb. 12-15) and Huancayo (Feb. 11-14).

Mean $\Delta H$ of these are $133 \gamma$ (instantaneous) for Losop and $138 \gamma$ (hourly mean) for Huancayo. M. Hasegawa and M. Ota [2], made detailed analysis of the Sq field for the II polar year data and concluded that a line of maximum diurnal magnetic variation (in place of which we write MS line in the following) is supposed, and lies nearly along the magnetic equator (dip zero line). A.T. Price [4] suggests, using the above mentioned data, that this line has some deviation from the magnetic equator. But according to Hasegawa's opinion, it is dangerous to decide this deviation from the above data, because observations near the magnetic equator have been very few, and direct observations in this region will be able to decide an exact deviation.

J. Egedal [5] showed the ranges for 1948-50 near the equator, his results are reproduced in Fig. 2 in which ratios of the ranges to that of Huancayo are shown for South America and India.
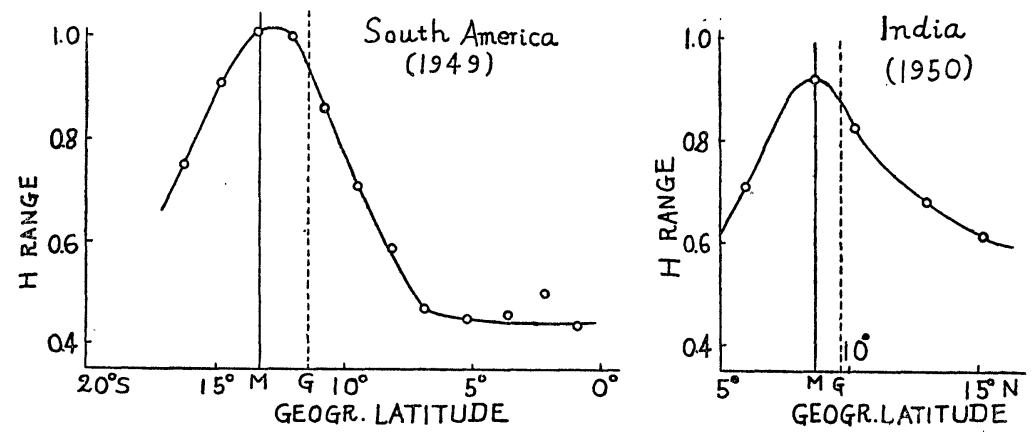

Fig. 2 Comparative Ranges of $H$ refered to Huancayo (After A.A. Giesecke and J. Egedal)

M : Magnetic Equator, G : Geomagnetic Equator (dipole equator)

From these results it is supposed that MS line in the Far East and in the Pacific seems to lie very closely on the magnetic equator. Mean ranges of $H$ at the magnetic equator shown by Egedal are as follows: South America 116 $\gamma$, India 106 $\gamma$, Togoland $124 \gamma$. These values are about as twice as that on the equator obtained by harmonic analysis. Magnitudes of main magnetic field are $0.3 \Gamma, 0.38 \Gamma, 0.32 \Gamma$ respectively for these places. South America and Togoland can hardly be compared because the latter's only is of instantaneous value. The range at India seems to be smaller than at South America and the main magnetic field of the former is greater than the latter's. At present it seems promising that MS line agrees with the magnetic equator, therefore we examine this fact theoretically.

\section{Influence of Hall Current}

In two previous papers [6] and [7], hereafter refered to as I and II respectively, we made preliminary calculations on the influence of the Hall Current (in place of which we write H.C, in the following) to the electrical conductivity of the ionosphere. 
The present paper describes a further examination of the problem.

To discuss conductivity in the ionosphere, it is necessary to consider a mixture of four gases--neutral molecules, positive ions, negative ions, and electrons. These are distinguished respectively by suffixes $a,+,-$ and $e$, and ions are denoted by $i$. The mass, charge (in e.m.u.), velocity, number density of $r$ th molecules are denoted by $m_{r}, e_{r}, \boldsymbol{c}_{r}, n_{r}$, and charge of an electron is specially denoted by $-e$. The number density, temperature of the gas as a whole are $n$ and $T$. The mass velocity $\boldsymbol{c}_{0}$ is defined by

$$
\boldsymbol{c}_{0}=\sum_{r} n_{r} m_{r} \overline{\mathbf{c}_{r}} / \sum_{r} n_{r} m_{r}
$$

and the peculiar velocity $\boldsymbol{C}_{r}$ is defined by $\boldsymbol{C}_{r}=\boldsymbol{c}_{r}-\boldsymbol{c}_{0}$. The gas as a whole is supposed to be almost electrically neutral;

thus

$$
\sum_{r} n_{r} e_{r} \simeq 0
$$

The electric current denșity $\boldsymbol{J}$ can be expressed in the form

$$
\boldsymbol{J}=\sum_{r} n_{r} e_{r} \overline{\boldsymbol{c}_{r}}=\sum_{r} n_{r} e_{r}\left(\boldsymbol{c}_{0}+\overline{\boldsymbol{C}_{r}}\right)=\sum_{r} n_{r} e_{r} \overline{\boldsymbol{C}_{r}}
$$

D.R. Bates [8] suggests that $n_{-} / n_{e} \equiv \lambda \ll 1$ in the $E$ and $F$ region. But this value in the $E$ region leaves some doubt, because reactions concerning molecular oxygen is not yet clear. T.G. Cowling [9], discussed the absorption of radio waves and suggested that $\lambda \ll 50$ in the $E$ region. According to Cowling, when $\lambda$ is not much greater than unity or when $n_{a} / n_{+}>5 \times 10^{5}$, the diffusion velocity $\overline{\boldsymbol{C}_{r}}$ can be expressed by the same form as for the free path method. In the ionosphere, the above conditions will be satisfied. When we discuss Sq current system, according to I $\S 1$, we get the next relation as a good approximation,

$$
\left\{\begin{array}{l}
\overline{\boldsymbol{C}_{r}}=\left(e_{r} / m_{r}\right)\left\{\nu_{r} /\left(\nu_{r}{ }^{2}+\omega_{r}{ }^{2}\right)\right\} \boldsymbol{E}_{\perp}-(1 / H)\left\{\omega_{r}{ }^{2} /\left(\nu_{r}{ }^{2}+\omega_{r}{ }^{2}\right)\right\} \boldsymbol{h} \times \boldsymbol{E}+\left(e_{r} / m_{r} \nu_{r}\right) \boldsymbol{E}_{l}(\gamma \neq a) \\
\overline{\boldsymbol{C}_{a}} \simeq 0
\end{array}\right.
$$

where $\boldsymbol{E}=\boldsymbol{E}^{(s)}+\boldsymbol{c}_{0} \times \boldsymbol{H}$ (in e.m.u.), $\boldsymbol{E}^{(s)}=$ electrostatic field, $\boldsymbol{H}=$ the earth's main magnetic field, $\boldsymbol{h}=$ unit vector in the direction of $\boldsymbol{H}, 1 / \nu_{r}=m_{r} D_{r} / k T, D_{r}=$ Diffusion coefficient between $r$ th molecule and neutral molecule. $\omega_{r}=e_{r} H / m_{r}, \boldsymbol{E}_{+}, \boldsymbol{E}_{l}=$ Component vectors of $\boldsymbol{E}$ perpendicular and parallel to the magnetic field. using (2), (3) and (4) we get

$$
\boldsymbol{J}=\sigma^{I} \boldsymbol{E}_{\perp}+\sigma^{I I} \cdot \boldsymbol{h} \times \boldsymbol{E}+\sigma_{\| /} \cdot \boldsymbol{E}_{\|},
$$

where

$$
\left\{\begin{array}{l}
\sigma^{I}=e^{2} \sum_{r}\left(n_{r} / m_{r}\right)\left\{\nu_{r} /\left(\nu_{r}^{2}+\omega_{r}{ }^{2}\right)\right\} \\
\sigma^{I I}=\left(n_{e} e / H\right)\left\{\omega_{e}{ }^{2} /\left(\nu_{e}{ }^{2}+\omega_{e}{ }^{2}\right)-\omega_{\imath}{ }^{2} /\left(\nu_{i}{ }^{2}+\omega_{i}{ }^{2}\right)\right\} \\
\sigma_{l}=e^{2} \sum_{r}\left(n_{r} / m_{r} \nu_{r}\right)
\end{array}\right.
$$

According to the dynamo theory, both horizontal tidal velocity $\boldsymbol{c}_{0}$ and the earth's magnetic field $\boldsymbol{H}$ in the ionosphere, produce primarily dynamo field $\boldsymbol{c}_{0} \times \boldsymbol{H}$, the vertical component of which is canceled by the resulting vertical electric field. The horizontal component of which produces a diurnal current system, together with a polarization field due to the resulting charge distribution. Near the $E$ region, when $\lambda \ll 1$ is satisfied, it is shown that $\sigma^{I} \sim 3 \times 10^{-21} n_{e}, \sigma^{I I} \sim 5 \times 10^{-20} n_{e}$, therefore $\sigma^{I I} / \sigma^{I} \sim 10$. Electric field produces not only a direct current, but also considerable H.C. which is shown by the 
second term of (5), therefore the dynamo theory may have to contain $\sigma^{I I}$ and differ from those of A. Schuster and S. Chapman. When $\lambda$ is not smaller than $1, \sigma^{I I} / \sigma^{I}$ becomes about $1 /(2 \lambda+1)$ times the above mentioned value, since $\sigma^{I}$ becomes about $(2 \lambda+1)$ times and $\sigma^{I I}$ is not increased. When $\lambda \gg 1$ is satisfied, we can neglect H.C., and even when $\lambda$ is small $\sigma^{I I} / \sigma^{I}$ rapidly decreases upward from the $E$ region. At first we treat the case that the axes of rotation and magnetism of the earth coincide.

Suppose that a uniform electric field is impressed in $\mathrm{E}-\mathrm{W}$ direction near the equator, then H.C. tends to flow vertically, but it cannot flow counteracted by a resulting polarization field; and as a resultant conductivity in $\mathrm{E}-\mathrm{W}$ direction, we get

which is shown in II, (10).

$$
\sigma^{I I I}=\sigma^{I}\left\{1+\left(\sigma^{I I} / \sigma^{I}\right)^{2}\right\}
$$

In this case positive and negative charges can move vertically by the same amount in the same direction. Let rectangular axis $\mathrm{O}$-xyz be taken, so placed that ox, oy, and $\mathrm{oz}$ are directed to the south, east, and upward respectively and unit vectors in the direction of ox, oy, and oz be $\boldsymbol{i}, \boldsymbol{j}$ and $\boldsymbol{k}$ respectively.

We treat first an ionosphere near the equator. Suppose that the layer is initially confined between $z=a_{1}$ and $z=a_{2}\left(a_{1}>a_{2}\right)$, electron density depends on $z$ only and is mainly controled by electron production and recombination, and that $\lambda$ is uniform, gas density distribution is the same as the real $E$ region. This treatment will be adequate, since the thickness of the $E$ region is several ten $\mathrm{kms}$ and the diurnal electric field is supposed to be approximately uniform over several thousand $\mathrm{kms}$. The charge density is denoted by $\rho$, the electric field is supposed to be perpendicular to the magnetic field, then we have the next equation in this layer

$$
\begin{aligned}
& \boldsymbol{J}=\sigma^{I} \cdot \boldsymbol{E}+\boldsymbol{\sigma}^{I I} \boldsymbol{h} \times \boldsymbol{E}, \\
& \nabla \cdot \boldsymbol{E}=4 \pi c^{2} \rho, \\
& \partial \rho / \partial t=-\nabla \cdot \boldsymbol{J}
\end{aligned}
$$

Suppose that constant electric field $E_{0}$ is impressed in oy direction from the time $t=0$, then H.C. produces polarization field $E_{z} \boldsymbol{k}$, and we get $\boldsymbol{E}=E_{\boldsymbol{l}} \boldsymbol{j}+E_{z} \boldsymbol{k}$. Substituting this for $\boldsymbol{E}$ in (7) we have $\quad J_{y}=\sigma^{I} \cdot E_{0}+\sigma^{I I} \cdot E_{z}, J_{z}=\sigma^{I} \cdot E_{z}-\sigma^{I I} \cdot E_{0}$.

As electron production and recombination are so rapid that variations of $\sigma^{I}$ and $\sigma^{I I}$ with current are negligible, the next equation is obtained $\partial J_{z} / \partial t=\sigma^{I} \cdot\left(\partial E_{z} / \partial t\right)$, on the other hand $\partial E_{z} / \partial t=-4 \pi c^{2} J_{z}$, therefore $\partial J_{z} / \partial t=-4 \pi c^{2} \sigma^{1} J_{z}\left(a_{2}<z<a_{1}\right) \therefore J_{z}=J_{z 0} e^{-t / \alpha}, \alpha=$ $1 / 4 \pi c^{2} \sigma^{I}$. As in the $E$ region we have $\sigma^{I} \sim 3 \times 10^{-21} n_{e}$ for gas density $n=10^{13}$ and $\lambda \leqslant 1$, therefore $\alpha \sim 3 \times 10^{-2} / n_{e}$. For so low an electron density $n_{e}=1$, follows $\alpha \sim 3 \times 10^{-2} \mathrm{sec}$. For $n_{e}=10^{5}$ we have $\alpha=3 \times 10^{-7} \mathrm{sec}$, but it is doubtful that we can treat the processes in such a short time by our present theory, since $1 / \nu_{i} \sim 2 \times 10^{-4}$ sec. at $n=10^{13}$. Nevertheless we can deduce that $J_{z}$ rapidly tends to zero after a time, say $2 \times 10^{-3}$ sec. When $\lambda$ is not smaller than $1, \alpha$ becomes about $1 /(2 \lambda+1)$ times the above value. Thus almost instantaneously, a steady state is attained and the relation $J_{z}=\sigma^{I} E_{z}-\sigma^{I I} E_{0}=0$ (11) holds at any height of the layer. At the same time from (10) $J_{y}=\sigma^{I}\left\{1+\left(\sigma^{I I} / \sigma^{I}\right)^{2}\right\} \cdot E_{0}$, therefore the conductivity of equation (6) is obtained at any height. Using equation 
(7) we get $\nabla \cdot \boldsymbol{J}=\sigma^{I} \nabla \cdot \boldsymbol{E}+\sigma^{I I} \cdot \nabla(\boldsymbol{h} \times \boldsymbol{E})+\nabla \sigma^{I} \cdot \boldsymbol{E}+\nabla \sigma^{I I} \cdot \boldsymbol{h} \times \boldsymbol{E}$. Writing $\sigma^{I}=n_{e} \cdot \sigma_{\nu}{ }^{I}, \sigma^{I I}=$ $n_{e} \cdot \sigma_{\nu}{ }^{I I}$, and considering equ. (8) and $\nabla \cdot(\boldsymbol{h} \times \boldsymbol{E})=-\partial E_{0} / \partial z=0$ we get the following equation

$$
-\partial \rho / \partial t=4 \pi c^{2} \cdot \sigma^{I} \cdot \rho+n_{e}\left\{E_{z}\left(\partial \sigma_{\nu}{ }^{I} / \partial z\right)-E_{0}\left(\partial \sigma_{\nu}{ }^{I} / \partial z\right)\right\}+\left(\sigma_{\nu}{ }^{I} \cdot E_{z}-\sigma_{\nu}{ }^{I I} \cdot E_{0}\right) \cdot \partial n_{e} / \partial z
$$

At the steady state, it follows from this equation that,

$$
\rho=\left(E_{0} \cdot \frac{\partial}{\partial z} \sigma_{\nu}{ }^{I}-E_{z} \cdot \frac{\partial}{\partial z} \sigma_{\nu}{ }^{I}\right) / 4 \pi c^{2} \cdot \sigma_{\nu}{ }^{I}
$$

This quantity depends on gas density and not on the electron density. In the $E$ region we infer that

$$
\begin{aligned}
& \sigma_{\nu}{ }^{T} \sim(2 \lambda+1) \cdot 1.76 \times 10^{-8} / n, \\
& \sigma_{\nu}{ }^{1 I} \sim(1 / H) 1.59 \times 10^{-20}\left(1-1.12 \times 10^{23} / n^{2}\right) .
\end{aligned}
$$

We take local scale height as $10 \mathrm{~km}$, then $\partial n / \partial z=10^{-6} \cdot n$. We have from (13), using these relation, $\rho=-E_{0} \cdot 9.03 \times 10^{-2 s}$ for $\lambda \ll 1$, and $n=10^{13}$. When solar tidal oscillations in this layer are $l_{s}$ times as great as those at the ground, we get $E_{0} \sim 3.7 l_{s}$ (e.m.u.) on the equator according to a later section, then we get

$$
\Delta n_{e}=\rho /(-e)=2.17 \times 10^{-7} \cdot l_{s} \text {. }
$$

As $l_{s}$ is estimated to be the order of $10^{2}, \Delta n_{e}$ is very small. Thus we can see how well the condition of electrical neutrality (2) is satisfied. When $\lambda \Varangle 1$ holds, we can also see the the same result. Next denote the total charge in a vertical column, which has a unit cross section on a plane $z=z_{1}$ and is confined in the range $z \leqq z_{1}$, by $q$, then we have at $z=z_{1}, E_{z}=4 \pi c^{2} q$. At the steady state, using (11) we obtain $q=$ $\left(1 / 4 \pi c^{2}\right)\left(\sigma^{I I} / \sigma^{I}\right) E_{0}=5.8 \times 10^{-21} l_{s}$ for $n=10^{13}$ at $z=z_{1}$. Let the length of the column be $d$, then from the above relation it follows that $d \cdot \Delta n_{e}=q / e=3.65 \times 10$ for $l_{s}=10^{2}$. For the value $d \sim 10 \mathrm{~km}$, we get $\Delta n_{e} \sim 3.65 \times 10^{-5}$.

If gas density in this layer is uniform, space charge distribution $\rho$ in the layer vanishes almost instantaneously and $E_{z}$ arises by a surface charge distribution $q$. From (11), as the mean value in the $E$ region, we get $\bar{E}_{z}=6 \cdot E_{0}$ for $\lambda \ll 1$.

\section{Conductivity at any Latitudes}

We treat the same the distribution of ionosphere as in the previous section. Here the magnetic field is supposed to be uniform with dip $\phi$ (constant). When a uniform electric field is impressed in either E-W direction or in N-S direction, we can show that the vertical current vanishes almost instantaneously on account of a vertical polarization field, but in this steady state, a vertical mass movement of the layer remains. When a uniform electric field $\left(\boldsymbol{E}_{x} \boldsymbol{i}+\boldsymbol{E}_{y} \boldsymbol{j}\right)$ is impressed, in the steady state we have

$$
\begin{aligned}
& J_{x}=\sigma_{x} E_{x}+\sigma_{x y} E_{y}, \quad J_{y}=\sigma_{y x} E_{x}+\sigma_{y} E_{y} \\
& E_{z}=\left\{\sigma^{I I} \cos ^{\prime} \phi \cdot E_{y}-\left(\sigma_{\sharp}-\sigma^{I}\right) \sin \phi \cos \phi \cdot E_{x}\right\} /\left(\sigma_{\eta} \cdot \sin ^{2} \phi+\sigma^{I} \cdot \cos ^{2} \phi\right)
\end{aligned}
$$

where

$$
\begin{aligned}
& \sigma_{x}=\sigma^{I} /\left\{\sin ^{2} \phi+\left(\sigma^{I} / \sigma_{n}\right) \cos ^{2} \phi\right\} \\
& \sigma_{x y}=\sigma^{I I} \sin \phi /\left\{\sin ^{2} \phi+\left(\sigma^{I} / \sigma_{\sharp}\right) \cos ^{2} \phi\right\} \\
& \sigma_{y x}=-\sigma_{x y} \\
& \sigma_{y}=\sigma^{I}+\left(\sigma^{I I} \cos \phi\right)^{2} /\left(\sigma^{I} \cos ^{2} \phi+\sigma_{n} \sin ^{2} \phi\right)
\end{aligned}
$$

The values of $\sigma_{x}$ and $\sigma_{y}$ were already discussed in I, $\S 3$ but $\sigma_{x y}$ in I, (17) is not valid 


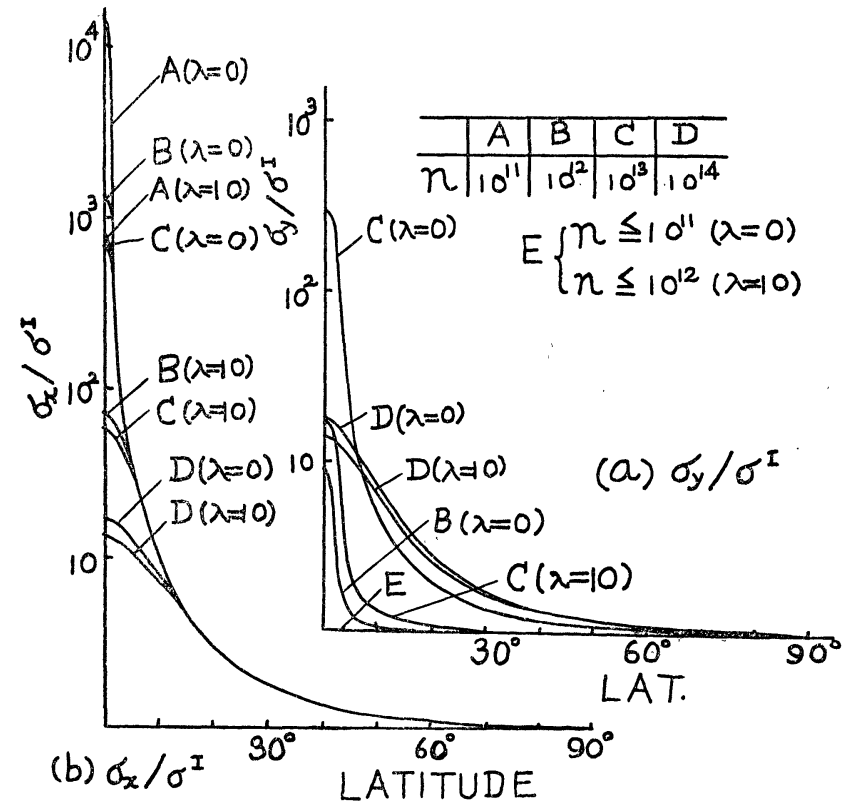

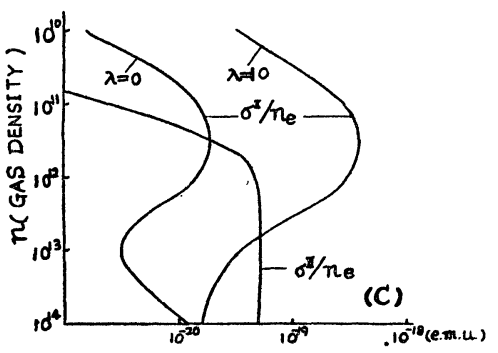

Fig. 3. The calculated values of (a) $\sigma_{y} / \sigma^{I}$, (b) $\sigma_{x} / \sigma^{I}$ with latitude, and (c) $\sigma^{I} / n_{e}, \sigma^{I I} / n_{e}$ with gas density.

since it does not take the vertical polarization field into consideration. The values of $\sigma_{y} / \sigma^{I}, \sigma_{x} / \sigma^{I}$ are illustrated with latitude in Figs 3 (a) and (b). $\sigma^{I} / n_{e}$ and $\sigma^{I I} / n_{e}$ with gas density are shown in Fig. 3 (c). Here we use the value by Ferraro [10] for $D_{i}$ with molecular weight 20 for ion and 25 for neutral molecule. $D_{e}$ is refered to gas kinetic value, and $H$ is taken to be 0.3 (e.m.u.) Therefore, we take the values $\nu_{e}=$ $1.31 \times 10^{-8} \cdot n, \nu_{i}=4.36 \times 10^{-10} \cdot n$. At middle latitudes $\sigma_{x} \sim \sigma^{I}$ and $\sigma_{y} \sim \sigma^{I}$. The above equations will be approximately valid, since both the magnetic field and diurnal electric field are supposed to be fairly uniform over far greater horizontal distance than the thickness of the ionosphere. To discuss the electrical conductivity of the $E$ region in higher latitudes than about $7 .^{\circ} 5$, it is necessary to consider $E$ and $F 1$ regions, which make tidal oscillations with air velocity $\boldsymbol{u}_{1} \boldsymbol{i}+v_{1} \boldsymbol{j}$ and $\boldsymbol{u}_{2} \boldsymbol{i}+v_{2: \boldsymbol{j}}$ respectively.

Let current density integrated with height in $E$ and $F \perp$ regions be $\boldsymbol{I}_{1}$ and $\boldsymbol{I}_{2}$, horizontal electrostatic field be $\boldsymbol{E}_{1}=X_{1} \boldsymbol{i}+Y_{1} \boldsymbol{j}$ and $\boldsymbol{E}_{2}=X_{2} \boldsymbol{i}+Y_{2} \boldsymbol{j}$. Then we get the following equations, for the $E$ region,

$$
\left\{\begin{array}{l}
I_{1 x}=\alpha_{x}\left(v_{1} H_{z}+X_{1}\right)+\alpha_{x y}\left(-u_{1} H_{z}+Y_{1}\right) \\
I_{1 y}=\alpha_{y x}\left(v_{1} H_{z}+X_{1}\right)+\alpha_{y}\left(-u_{1} H_{z}+Y_{1}\right)
\end{array}\right.
$$

for the $F 1$ region,

$$
\left\{\begin{array}{l}
I_{2 x}=\beta_{x}\left(v_{2} H_{z}+X_{2}\right) \\
I_{2 y}=\beta_{y}\left(-u u_{2} H_{z}+Y_{2}\right)
\end{array}\right.
$$

where $\alpha_{r}=\int_{E} \sigma_{r} d h \quad(\gamma=x, x y, y x, y), \quad \beta_{r}=\int_{F 1} \sigma_{r} d h, \quad \beta_{x y}, \beta_{y x}$ are effectively zero. As in the usual dynamo theory, the relation $\boldsymbol{\nabla} \cdot \boldsymbol{J} \simeq 0$ can be readily seen here.

If we take the $F$ region in place of the $F 1$, the values of $\beta_{r}$ will be a little increased. 
An icieal case that these two layers are electromagnetically independent, was first raised by Dr. K. Maeda [11].

But we think that interactions of these layers are important. At present it is most probable that the $\mathrm{Sq}$ current flows mainly in the $E$ region. We imagine the following model for Sq field in the daytime. In the northern hemisphere, anticlockwise electric field (dynamo field plus electrostatic field) of a whirl shape will be present. Direct curect will flow along this field as shown by I, Fig. 4. Horizontal component of H.C. will flow in such a way as to concentrate negative charges to the focus of the whirl. This polarization will, however, be considerably dissipated by direct current in the $F$ region in the opposite direction to the lower horizontal H.C.; since in higher latitudes than about $7 .^{\circ} 5$, as shown in II, the $E$ region is connected by highly conductive lines of magnetic force to the $F$ region. Therefore the effective conductivity of the $E$ region in these latitudes will be much less than that at the magnetic equator.

In the following we suppose that $\lambda \ll 1, E$ and $F 1$ layers are thin spherical shells with the above mentioned conductivities, and their distances are so small that they can be represented by a common radius $a$ in mathematical treatment.

Let $E$ and $F \perp$ layers be Chapman layers with local scale height $10 \mathrm{~km}$ and $30 \mathrm{~km}$, gas ciensities at these centers be $6 \times 10^{12}$ and $10^{11}$, and maximum electron densities $10^{5} / \mathrm{cc}$ and $2.5 \times 10^{5} / \mathrm{cc}$. For this model, we obtain a distribution of $\sigma_{\|}$with height. Between $E$ and $F 1$ layers we obtain by a numerical calculation, $1 / \overline{\left(1 / \sigma_{\eta}\right)}=7.95 \times 10^{-13}$. (e.m.u.) which is very great compared with the values at respective centers, $\sigma^{I}(E)=$ $4.4 \times 10^{-16}$ and $\sigma^{I}(F 1)=3.58 \times 10^{-15}$. Therefore we can show that $E$ and $F 1$ regions are connected by highly conductive lines of force in middle latitudes. But vertical current will be very small. Here we assume that Sq current system is hearly horizontal, i.e. $J_{z} \ll V \overline{J_{x}{ }^{2}+J_{y}{ }^{2}}$. Considering the relation $\sigma_{n} \gg \sigma^{I}, \sigma^{I I}$, equation (15) can be reduced to

$$
E_{z} \simeq-\cot \phi \cdot E_{x} \text {. }
$$

On the other hand, as the third term of equation (5) can be written as

$$
\sigma_{\|} \boldsymbol{E}_{\eta}=\sigma_{\eta}\left(-E_{x} \cos \phi-E_{z} \sin \phi\right) \boldsymbol{h}
$$

equation (19) implies that $\boldsymbol{E}_{\eta}$ is very small. Therefore, if $\boldsymbol{E}_{z}$ deviates a little from the value of (15), current flows almost in the direction of lines of magnetic field, and the above assumption is violated. Thus equation (15) and consequently (14), (16), (17) and (18) hold with good approximation, in this case. As the relation between $E_{x}, E_{y}$, and $E_{z}$ in the equation (15) is linear, we can divide $E_{2}$ into two parts; the one produced by the horizontal dynamo field, and the other produced by the horizontal electrostatic field. These sets satisfy the equation (15) respectively. In the following we treat the latter set. Let a vertical square $a b c d$ be taken, as shown in Fig. 4 , so placed that $a b$ and $c d$ are horizontal and situated at the center of $E$ and $F 1$ region, and in the direction of electric field in a practical sense, and that this square is fixed to the earth. Horizontal electrostatic fields on $a b$ and $c d$ be $E_{h}$ and $E_{h}^{\prime}$, which are measured positively in the direction of $\overrightarrow{a b}$, upward vertical component of electrostatic fields on $b c$ and $a d$ be $E_{z}$ and $E_{z}^{\prime}$. 


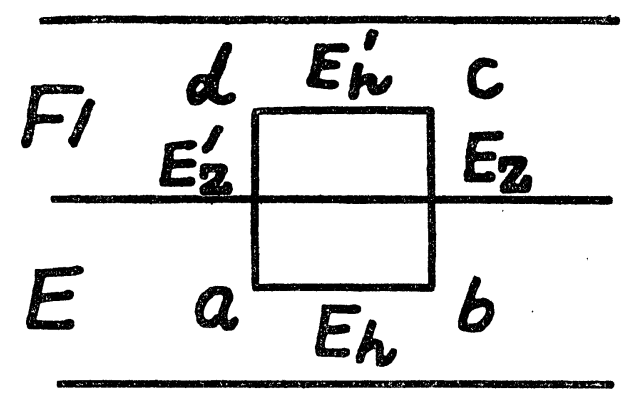

Fig. 4

As $\oint \boldsymbol{E} d \boldsymbol{s}=0$ around $a b c d$, we obtain the following equation

$$
\overline{E_{h}}-{\overline{E^{\prime}}}_{h}=\overline{E_{z}^{\prime}}-\overline{E_{z}}
$$

where bar denotes mean value on each side of the square.

Let a length of one side be $L$, which is of the order of $10^{7} \mathrm{~cm}$. Direction cosine of $\overrightarrow{a b}$ be $(l, m, 0)$, then differential coefficient

in the direction of $a b$ can be written, using equation (19) as

$$
d E_{z} / d s=-l\left(E_{x} \cdot \partial \cot \phi / a \partial \theta+\cot \phi \cdot \partial E_{x} / a \partial \theta\right)-m\left(\cot \phi \cdot \partial E_{x} / a \sin \theta \partial \lambda\right)
$$

We deduce, from the observed results, that

$$
\partial E_{x} / a \partial \theta \sim E_{x} / a, \quad \partial E_{x} / a \sin \theta \partial \lambda \sim E_{x} / a
$$

Therefore $\quad \partial E_{z} / \partial s=-\left(E_{x} / a\right)\left[l\left\{2 / \sin ^{2} \phi\left(1+3 \cos ^{2} \theta\right)+\cot \phi\right\}+m \cot \phi\right]$

Under the assumption that horizontal electric field in every region is of the same order of magnitude as that at the center of the region, and considering that $\overline{E_{z}^{\prime}}-\overline{E_{z}}$ $\simeq\left(d \bar{E}_{z} / d s\right) L$, we can make the following estimation. In higher latitudes than $20^{\circ}$,

$$
\begin{aligned}
& \quad\left|\overline{E_{i l}}-{\overline{E^{\prime}}}_{h}\right| \leqq\left\{\left|{\overline{E_{h}}}_{1}+\right|{\overline{E^{\prime}}}_{h} \mid\right\} \cdot\left|l\left(l \cdot \frac{1}{30}+m \cdot \frac{1}{80}\right)\right| \\
& \therefore \quad\left|\overline{E_{h}}-{\overline{E^{\prime}}}_{h}\right| \ll\left|\bar{E}_{h}\right|+\left|{\overline{E^{\prime}}}_{h}\right| .
\end{aligned}
$$

This relation holds approximately near $7 .^{\circ} 5$ but does not hold in lower latitudes if $\overrightarrow{a b}$ is in the N-S direction. Therefore in higher latitudes than $7 .{ }^{\circ} 5$ we can take $\bar{E}_{h}=\bar{E}_{h}^{\prime}$ as a first approximation. Thus we write $X_{1}=X_{2}=X, Y_{1}=Y_{2}=Y$ in (17) and (18).

The above result will be approximately valid for $E$ and $F$ region. For such a uniform current system as Sq, difference of the height of current sheet, of $100 \mathrm{~km}$, gives negligible difference to the variation of terrestrial magnetism at the ground. Therefore, Sq is produced by $\boldsymbol{I}=\boldsymbol{I}_{1}+\boldsymbol{I}_{2}$ in a good approximation. We can, further, treat in such a way that $\partial I_{x} / a \partial \theta+\partial I_{y} / a \sin \theta \partial \lambda=0$, to be derived from $\boldsymbol{V} \cdot \boldsymbol{J}=0$, considering $J_{z}=0$ on the outer surfaces of the combined layer.

Then the following two cases are examined.

(I) $F$ region is at rest i.e. $u_{2}=v_{2}=0$ and $E$ region makes tidal oscillations.

We write $\boldsymbol{I}=\boldsymbol{I}_{x} \boldsymbol{i}+\boldsymbol{I}_{y} \boldsymbol{j},\left(\boldsymbol{I}_{z} \ll I_{x}, \boldsymbol{I}_{y}\right)$, and from (17) and (18), get

$$
\left\{\begin{array}{l}
I_{x}=\alpha_{x x}\left(v_{1} H_{z}+X\right)+\alpha_{x y}\left(-u_{1} H_{z}+Y\right)+\beta_{x} X \\
I_{x}=\alpha_{y x}\left(v_{1} H_{z}+X\right)+\alpha_{y}\left(-u_{1} H_{z}+Y\right)+\beta_{y} Y
\end{array}\right.
$$

$X \boldsymbol{i}+Y \boldsymbol{j}$ can be derived from a potential $S$, i.e. $X=-\partial \boldsymbol{S} / a \partial \theta, Y=-\partial S / a \sin \theta \partial \lambda$.

Substituting these values in (22) and by the eiimination of $S$, we have

$$
\begin{aligned}
\frac{\partial}{\partial \lambda}\left(\frac{1}{K_{x}} I_{x}\right)-\frac{\partial}{\partial \lambda} & \left(\frac{1}{K_{x y}} I_{y}\right)-\frac{\partial}{\partial \theta}\left(\frac{\sin \theta}{K_{y}} I_{y}\right)+\frac{\partial}{\partial \theta}\left(\frac{\sin \theta}{K_{y x}} I_{x}\right) \\
& =\frac{\partial}{\partial \lambda}\left\{H_{z}\left(M_{1} u_{1}+M_{2} v_{1}\right)\right\}+\frac{\partial}{\partial \theta}\left\{H_{z} \sin \theta\left(M_{3} u_{1}+M_{4} v_{1}\right)\right\}
\end{aligned}
$$


where

$$
\begin{aligned}
& K_{x}=\left(\alpha_{x}+\beta_{x}\right)+\frac{\alpha_{x y}{ }^{2}}{\alpha_{y}+\beta_{y}}, \quad K_{y}=\left(\alpha_{y}+\beta_{y}\right)+\frac{\alpha_{x y} y^{2}}{\alpha_{x}+\beta_{x}}, \\
& K_{x y}=\alpha_{x y}+\left(\alpha_{x}+\beta_{x}\right)\left(\alpha_{y}+\beta_{y}\right) / \alpha_{x y}, \quad K_{y x}=-K_{x y}, \\
& M_{1}=-\left(\beta_{y} \cdot \alpha_{x y}\right) / \Delta, \quad M_{2}=\left\{\alpha_{x}\left(\alpha_{y}+\beta_{y}\right)+\alpha_{x y}{ }^{2}\right\} / \Delta, \\
& M_{3}=\left\{\left(\alpha_{x}+\beta_{x}\right) \alpha_{y}+\alpha_{x y}{ }^{2}\right\} / \Delta, \quad M_{4}=\left(\beta_{x} \cdot \alpha_{x y}\right) / \Delta, \\
& \text { with } \quad \Delta=\left(\alpha_{x}+\beta_{x}\right)\left(\alpha_{y}+\beta_{y}\right)+\alpha_{x y}{ }^{2}
\end{aligned}
$$

Here we assume that electron density is constant at the same altitude. At the latitudes, here concerned, we get the following values approximately

$$
\begin{cases}K_{x}=K_{1} / \sin ^{2} \phi, & K_{y}=K_{1}=\left(\alpha^{I}+\beta^{I}\right)+\left(\alpha^{I I}\right)^{2} /\left(\alpha^{I}+\beta^{I}\right) \\ K_{x y}=K_{0} / \sin \phi, & K_{0}=\alpha^{I I}+\left(\alpha^{I}+\beta^{I}\right)^{2} / \alpha^{I I}\end{cases}
$$

where $\boldsymbol{a}^{T}=\int_{E} \sigma^{T} d h$ etc. $(\phi=\mathrm{Dip})$.

$\begin{array}{ll}M_{1}=-\gamma_{1} / \sin \phi, \quad M_{2}=\gamma_{2}, \quad M_{3}=\gamma_{2}, \quad M_{4}=\gamma \\ \text { where } & \gamma_{1}=\beta^{I} \cdot \alpha^{I I} / G, \quad \gamma_{2}=\left\{\alpha^{I}\left(\alpha^{I}+\beta^{I}\right)+\left(\alpha^{I I}\right)^{2}\right\} / G,\end{array}$

with $\quad G=\left(\alpha^{I}+\beta^{I}\right)^{2}+\left(\alpha^{I I}\right)^{2}$

$K_{1}, K_{0}, \gamma_{1}, \gamma_{2}$ are independent of $\lambda$ and $\theta$, if we neglect very slow variations depending on the magnitude of the magnetic field. Let velocity potential of the air be

$$
\Psi_{\sigma}^{\sigma}=k_{\sigma}^{\sigma} P_{\sigma}^{\sigma} \sin \left(\sigma t+\sigma_{\sigma}^{\sigma}\right)
$$

where $k_{\sigma}^{\sigma}$ is taken to be positive, $t=t^{\prime}+\lambda$ denotes local time, $P_{m}^{n}$ denotes associated Legendre function of Neumann.

Then $\quad u_{1}=\partial \psi_{\sigma}^{\sigma} / a \partial \theta, \quad v_{1}=\partial \psi_{\sigma}^{\sigma} / a \sin \theta \partial \lambda$.

We write $H_{z}=C \cos \theta(C<0)$, and the right hand side of equation (23) is reduced to the form,

$$
-\frac{1}{a} \cdot C \cdot k_{\sigma}^{\sigma} \sin \theta\left\{\frac{\gamma_{2}(\sigma+2) \sigma}{2 \sigma+1} P_{\sigma+1}^{\sigma} \sin \left(\sigma t+\sigma_{\sigma}^{\sigma}\right)+\gamma_{1} \cdot \sigma \Phi(\theta) P_{\sigma}^{\sigma} \cos \left(\sigma t+\alpha_{\sigma}^{\sigma}\right)\right\}
$$

where

$$
\Phi(\theta)=3 \cos ^{2} \theta /\left(1+3 \cos ^{2} \theta\right) \cdot \sin \phi
$$

We shall treat, mainly the semi-diurnal air velocity $(\sigma=2)$. For numerical example, two cases (a), and (b) are considered.

Case (a)

$$
\alpha^{I}=5 \times 10^{-9}, \quad \alpha^{I I}=3 \times 10^{-8}, \quad \beta^{I}=4 \times 10^{-8} \text { (e.m.u.) }
$$

using these values we get

$$
K_{1}=13 \alpha^{I}, \quad K_{0}=19.5 \alpha^{I} ; \quad \gamma_{1}=0.41, \quad \gamma_{2}=0.385
$$

Case (b)

$$
\alpha^{I}=5 \times 10^{-9}, \quad \alpha^{I I}=3 \times 10^{-8}, \quad \beta^{I}=1.5 \times 10^{-8}
$$

using these values we get

$$
K_{1}=13.6 \alpha^{I}, \quad K_{0}=8.16 \alpha^{I} ; \quad \gamma_{1}=0.346, \quad \gamma_{2}=0.77
$$

These values of case (a) are obtained in II. If Bates and Massey's [17] estimation of gas density depending on rocket measurements, are correct, then case (b) will be better. At present the effective conductivity of the $F$ layer to the electrostatic field is not yet clear and these examples will give rough order of magnitude to the following calculation.

The values of $\left|\frac{\gamma_{2}(\sigma+2) \sigma}{2 \sigma+1} P_{\sigma+1}^{\sigma}\right|$ and $\left|\gamma_{1} \cdot \sigma \cdot \emptyset(\theta) P_{\sigma}^{\sigma}\right|$, for $\sigma=2$ and case (a), are 


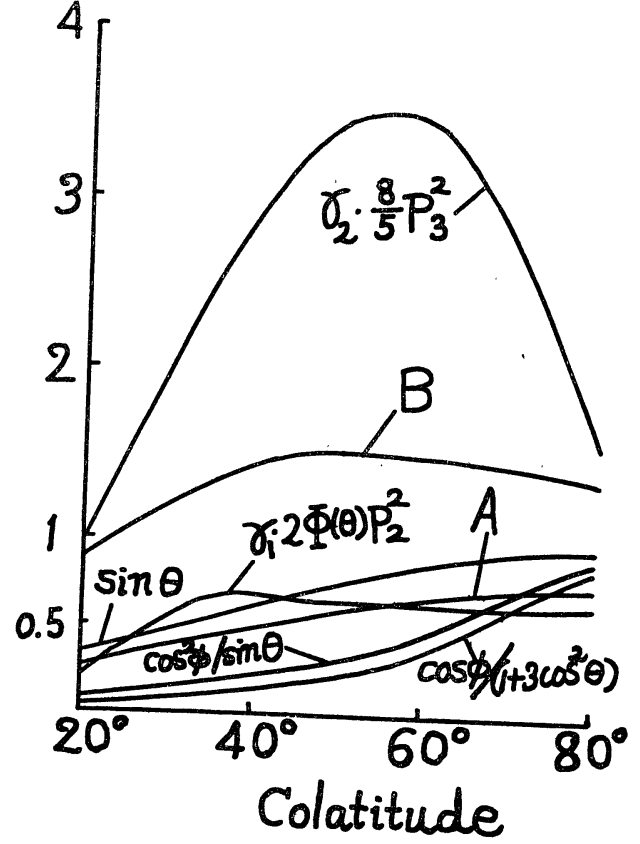

Fig. 5 The Values of $\gamma_{2} \cdot(8 / 5) P_{3}{ }^{2}, \gamma_{1} \cdot 2 \Phi(\theta) P_{2}{ }^{2}$; $\sin \theta, \cos ^{2} \phi / \sin \theta, \cos \phi /\left(1+3 \cos ^{2} \theta\right)$; and $A, B$ of the equation (38), with colatitude.

shown with colatitude $\theta$, in Fig. 5. As the latter's is much less than the former's, we can neglect the second term of (25), to discuss the order of magnitude. For case (b) we obtain the same result.

Since $\boldsymbol{\nabla} \cdot \boldsymbol{I}=0, \boldsymbol{I}$ cán be derived from a current function $R$, i.e.

$$
I_{x}=\partial R / a \sin \theta \partial \lambda, \quad I_{y}=-\partial R / a \partial \theta .
$$

After substitution, of these values, the left hand side of equ. (23) is reduced to the next form,

$$
\begin{aligned}
& \frac{1}{a K_{1}}\left[\left\{\frac{\partial^{2} R}{\sin \theta \partial \lambda^{2}}+\frac{\partial}{\partial \theta}\left(\sin \theta \cdot \frac{\partial R}{\partial \theta}\right)\right\}\right. \\
& \left.-\cos ^{2} \phi \cdot \frac{\partial^{2} R}{\sin \theta \partial \lambda^{2}}\right]+\frac{1}{a K_{0}} \cdot \frac{2 \cos \phi}{1+3 \cos ^{2} \theta} \cdot \frac{\partial R}{\partial \lambda}
\end{aligned}
$$

Suppose that the current function $R$, to be determined from (23), is expressed by a tesseral harmonic, $R=r_{n}^{m} P_{n}^{m} \sin \left(m t+\sigma_{n}^{m}\right)$.

By substitution of this value, (26) is reduced to the form,

$$
\begin{gathered}
\frac{1}{a K_{1}}\left\{-\sin \theta \cdot n(n+1) r_{n}^{m} P_{n}^{m} \sin \left(m t+\alpha_{n}^{m}\right)+\frac{\cos ^{2} \phi}{\sin \theta} \cdot m^{2} \cdot r_{n}^{m} P_{n}^{m} \sin \left(m t+\sigma_{n}^{m}\right)\right\} \\
+\frac{1}{a K_{0}} \cdot \frac{\cos \phi}{1+3 \cos ^{2} \theta} \cdot 2 m \cdot r_{n}^{m} P_{n}^{m} \cos \left(m t+\alpha_{n}^{m}\right)
\end{gathered}
$$

As we are treating semi-diurnal variations, $m=2$, and moreover $n \geqq 3$, since the current must be symmetrical against the equator. The values of $\sin \theta, \cos ^{2} \phi / \sin \theta, \cos \phi /(1+$ $\left.3 \cos ^{2} \theta\right)$ are shown in Fig. 5 with colatiude. From these results we can eliminate the second term of (28) against the first term. Further, considering $n(n+1) / K_{1} \geqq 0.925 / \boldsymbol{\alpha}^{I}$ and $2 m / K_{0}=0.205 / \alpha^{I}$ for case (a), we can neglect the third term of (28). Thus (28) can be expressed by the first term neglecting an error of the order of about $30 \%$. For case (b), we can obtain similar results.

Hence equation (23) can be recuced to the equation,

$$
\sin \theta \cdot n(n+1) r_{n}^{m} P_{n}^{m} \cdot \sin \left(m t+\alpha_{n}^{m}\right)=C \cdot K_{e} \cdot k_{\sigma}^{\sigma} \sin \theta \cdot \frac{(\sigma+2) \sigma}{2 \sigma+1} P_{\sigma+1}^{\sigma} \sin \left(\sigma t+\alpha_{\sigma}^{\sigma}\right)
$$

where $\quad K_{e}=K_{1} \cdot \gamma_{2}$

Therefore,

$$
R=\gamma_{\sigma+1}^{\sigma} \cdot P_{\sigma+1}^{\sigma} \sin \left(\sigma t+\alpha_{\sigma}^{\sigma}\right)
$$

where

$$
r_{\sigma+1}^{\sigma}=C \cdot K_{e} \cdot k_{\sigma}^{\sigma} \sigma /(\sigma+1) \cdot(2 \sigma+1) .
$$

On the other hand, when the spherical shell has direct, isotropic, constant conductivity $K$ only, dynamo equations for this case are written as 


$$
\left.\begin{array}{c}
v_{1} H_{z}-\frac{\partial S}{a \partial \theta}=\frac{1}{K} \frac{\partial R}{a \sin \theta \partial \lambda} \\
-u_{1} H_{z}-\frac{\partial S}{a \sin \theta \partial \lambda}=-\frac{1}{K} \frac{\partial R}{a \partial \theta}
\end{array}\right\}
$$

From these two equations, by eliminating $S$, and substituting (27) for $R$, and $K_{e}$ for $K$, the same equation as (29) is obtained. Hence $K_{e}$ is interpreted as an effective conductivity to the dynamo current in these latitudes. $K_{e}$ is represented as

$$
K_{e}=\boldsymbol{\alpha}^{I}+\left(\boldsymbol{\alpha}^{I I}\right)^{2} /\left(\boldsymbol{\alpha}^{I}+\beta^{I}\right),
$$

and $K_{e}=5 \alpha^{I}$ for case (a), $K_{e}=10 \alpha^{I}$ for case (b).

(II) $E$ and $F 1$ regions make tidal oscillations with the same velocity i.e. $u_{2}=u_{1}, v_{2}=v_{1}$.

In place of equation (22) we obtain

$$
\left\{\begin{array}{l}
I_{x}=\left(\alpha_{x}+\beta_{x}\right)\left(v_{1} H_{z}+X\right)+\alpha_{x y}\left(-u_{1} H_{z}+Y\right) \\
I_{y}=\alpha_{y x} \cdot\left(v_{1} H_{z}+X\right)+\left(\alpha_{y}+\beta_{y}\right)\left(-u_{1} H_{z}+Y\right)
\end{array}\right.
$$

From these equations we have, in place of (23),

$$
\begin{aligned}
\frac{\partial}{\partial \lambda}\left(\frac{1}{K_{x}} I_{x}\right)-\frac{\partial}{\partial \lambda}\left(\frac{1}{K_{x y}} I_{y}\right)-\frac{\partial}{\partial \theta}\left(\frac{\sin \theta}{K_{y}} I_{y}\right)+\frac{\partial}{\partial \theta}\left(\frac{\sin \theta}{K_{y x}} I_{x}\right) \\
=\frac{\partial}{\partial \lambda}\left(v_{1} H_{z}\right)+\frac{\partial}{\partial \theta}\left(\sin \theta \cdot u_{1} H_{z}\right)
\end{aligned}
$$

The left hand side of this equation can be reduced in the same way as before, and the right hand side is reduced without any neglection, to the first term of (25), in which 1 is substituted for $\gamma_{2}$. By the same deduction as before, effective conductivity $K_{1}$ is obtained. The value of $K_{1}$ is $K_{1}=K_{e}+\beta^{I}$. Therefore, in this case also, effective conductivity of the $E$ layer is considered to be $K_{e}$.

\section{Sq current near the Magnetic Equator}

In a narrow belt along line of zero dip of several degrees wide, the equation (19) may not hold, since both current and magnetic fields are nearly horizontal. The $\sigma_{y x}$ in the equation (14) will not be valid and $\sigma_{y_{x}}$ in I, (17) might be better and the difference of these values is very great. At present we have not been able to find the best value of $\sigma_{y x}$. Thus, we treat this region rather in the same way as in section 2.

In this belt in the $E$ region, we have

$$
I_{1 x} \simeq \alpha_{n} X_{1}, \quad I_{1 y} \simeq \alpha^{I 1 I} \cdot Y_{1}
$$

where $\quad \boldsymbol{\alpha}^{I I I}=\int_{E} \sigma^{I I I} d h, \quad \boldsymbol{\alpha}_{n}=\int_{E} \sigma_{\eta} d h$.

In this belt, in the $F 1$ region, $\quad I_{2 x} \simeq \beta_{\eta} X_{2}, \quad I_{2 y} \simeq \beta^{I} Y_{2}$.

By the same way as in middle latitudes, we can show that $Y_{1} \simeq Y_{2}$, but we have $X_{1} \neq X_{2}$. Hence effective conductivity in E-W direction will be $\alpha^{I I I}+\beta^{I}$ and that in $\mathrm{N}$-S direction will be a certain average of $\alpha_{n}$ and $\beta_{l}$. The latter will be larger than the former, since $\sigma_{\|}$is much greater than $\sigma^{I}, \sigma^{I I}$ and $\sigma^{I I I}$. As the most resistant part is effective to the dynamo current, $\alpha^{I I I}+\beta^{I}$ will be the effective conductivity in this belt. 
For case (a)

$$
\alpha^{I I I}+\beta^{I}=45 \alpha^{I}, \quad\left(\alpha^{I I I}+\beta^{I}\right) / K_{1}=3.5, \quad \alpha^{I I I} / K_{e}=7.5
$$

and for case (b)

$$
\alpha^{I I I}+\beta^{I}=39.6 \alpha^{I}, \quad\left(\alpha^{I I I}+\beta^{I}\right) / K_{1}=3, \quad \alpha^{I I I} / K_{e}=3.7 .
$$

For any case, effective conductivity in this belt is greater than 3 times that in middle latitudes. This conductivity will slowly decrease from the equator towards 7.05 of latitudes. Therefore, to see the outline of the Sq current distribution, we may use the dynamo equation (31) for the following distribution of $K$, (A) $K=K_{L}$ (const.) for $0 \leqq \theta<\theta_{1} \quad$ (B) $K=\nu K_{L}$ for $\theta_{1}<\theta<\pi-\theta_{1} \quad$ (C) $K=K_{L}$ for $\pi-\theta_{1}<\theta \leqq \pi, \theta_{1}=85^{\circ}$ is used in this section. From equation (31), by eliminating $S$ we obtain

$$
\frac{\partial^{2} R}{\sin \theta \partial \lambda^{2}}+\frac{\partial}{\partial \theta}\left(\sin \theta \cdot \frac{\partial R}{\partial \theta}\right)=a K\left\{\frac{\partial\left(v H_{z}\right)}{\partial \lambda}+\frac{\partial\left(u H_{z} \sin \theta\right)}{\partial \theta}\right\}
$$

First, we consider the solution of the equation

$$
\frac{\partial^{2} R}{\sin \theta \partial \lambda^{2}}+\frac{\partial}{\partial \theta}\left(\sin \theta \cdot \frac{\partial R}{\partial \theta}\right)=0
$$

Substituting $R=f(\theta) \cdot F(\lambda)$ in the above, we get equations (20) and (21) in II. The solution of (21) in II is denoted by $Q_{0}{ }^{M}$ by Hobson [13]. Using his formula, an integral of (36) is expressed as

$$
\sum_{M}\left\{A_{M}\left(1-\mu^{2}\right)^{\frac{1}{2} M} /(1-\mu)^{M}+B_{M}\left(1-\mu^{2}\right)^{\frac{1}{2} M} /(1+\mu)^{M}\right\}\left(D_{M} \sin M \lambda+E_{M} \cos M \lambda\right),
$$

where $M$ is an integer, $\mu=\cos \theta$, and $A_{M}, B_{M}, D_{M}, E_{M}$ are integrating constants. A general value of the integral of (35) is obtained as the sum of a particular integral of (35) and the above integral. Thus, for semi-diurnal variation we obtain the following solution

$$
\begin{array}{ll}
\text { (A) } & R=B \cdot K_{L}\left\{P_{3}{ }^{2}(\theta)+C_{1} q_{1}(\theta)\right\} \sin (2 t+\alpha) \\
\text { (B) } & R=B \nu K_{L}\left\{P_{3}{ }^{2}(\theta)+C_{2} q_{1}(\theta)+C_{3} q_{2}(\theta)\right\} \sin (2 t+\alpha) \\
\text { (C) } & R=B \cdot K_{L}\left\{P_{3}{ }^{2}(\theta)+C_{4} q_{2}(\theta)\right\} \sin (2 t+\alpha)
\end{array}
$$

where $B=(2 / 15) C k_{2}{ }^{2}, \quad q_{1}(\theta)=(1-\mu) /(1+\mu), \quad q_{2}(\theta)=(1+\mu) /(1-\mu)$

Considering the continuity of the normal component of the current and the tangential component of the electrostatic field along the boundary between (A), (B) and (B), (C),
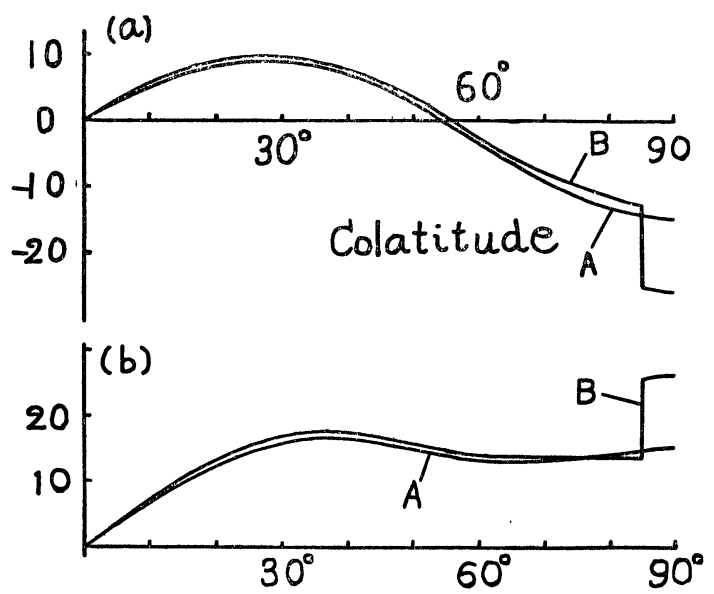

Fig. 6 (a) $J_{0}(\theta)$, (b) Amplitude of Current Density in unit of $\left(-2 C k_{2}^{2} K_{L} / 15 a\right)$ we determine $C_{1}, C_{2}, C_{3}, C_{4}$. Eastward current $J_{y}$ is obtained by the following equation $J_{y}=-\partial R / a \partial \theta=J_{0}(\theta) \sin (2 t+\boldsymbol{\alpha})$, $J_{0}(\theta) /\left(-B K_{L}\right)$ with colatitude is shown in Fig. 6 (a). Curve $A$ denotes the case $\nu=1$ and curve $B$ the case $\nu=2$ both for $\theta_{1}=85^{\circ}$. Amplitude of $\boldsymbol{J}$ is shown in Fig. 6 (b). Thus we can show MS line by the line of maximum amplitude of $\boldsymbol{J}$. When solar tidal oscillations in the ionosphere are $l_{s}$ times as great as those at the ground, according to Chapman [14], we get $k_{2}{ }^{2} \sim 5 l_{s} a$. 


\section{Vertical Drift Velocity of F2 Layer produced by the Hall Current}

In this section we discuss the case that only $E$ region makes tidal oscillations: In section 3 , we showed that $\boldsymbol{E}_{1} \simeq \boldsymbol{E}_{2}$, and this result will be approximately valid for $E$ and $F$ region. Since this conclusion depends upon some assumption and deduction, it is clearly desirable to submit it to a direct test. It has been shown above that when we discuss semi-diurnal variation, in higher latitudes than $7 .^{\circ} 5$, the current function $R=r_{3}{ }^{2} P_{3}{ }^{2} \sin (2 t+\alpha), \alpha=\alpha_{2}{ }^{2}$ is a fairly good approximation for the current.

From equations (22) and the above value we obtain

$$
\begin{gathered}
Y=(1 / a) \cdot C \cdot{k_{2}}^{2}\left\{A \sin (2 t+\alpha)+B \sin \left(2 t+\alpha+90^{\circ}\right)\right\} \\
=(1 / a) \cdot C \cdot{k_{2}}^{2} \cdot D \sin (2 t+\alpha+\varphi), \\
A=\left(K_{e} / K_{1}\right)\left\{(-2 / 15) \partial P_{3}{ }^{2} / \partial \theta+\cos \theta \cdot \partial P_{2}{ }^{2} / \partial \theta\right\} \\
B=(4 / 3)\left(K_{e} / K_{0}\right) \sin \phi \cdot P_{2}{ }^{1}+(2 / \sin \phi) \cdot P_{2}{ }^{1} \cdot\left(\alpha^{I I} / K_{1}-\alpha_{2}{ }^{I} / K_{0}\right)
\end{gathered}
$$

where

In $F 2$ region the second term of equation (4) is the same for ions and electrons, and a vertical component $W$ of this term represents the vertical drift of this region. Thus, we obtain

$$
W=\frac{\cos \phi}{H_{0} V} \cdot Y
$$

D.F. Martyn [15] suggested that main seat of the lunar current is the $D$ region. Later, it was pointed out by Cowling and Borger [16], that conductivity of the $D$ region is too low to be a main seat. The same objection was stressed by Bates and Massey [17]. Though the $F$ region has considerable conductivity, induction drag [9] and kinematic viscosity are very effective in this region. Tidal motion in this region may be very small, from the theoretical point of view. Therefore, it will be possible that lunar main current flows in the $E$ region. Suppose that lunar main current flows in the $E$ region, then $\alpha=-17^{\circ}$ should be satisfied to agree with Chapman's results for 1905 equinox (14).

If Hall Current is not taken into account, $Y$ is of the same phase as the first term of equation (38), then $W$ is almost downward during 6 hours after lunar transit. While as regards world wide lunar $F 2$ variation, except very near the magnetic equator, amplitudes of oscillation of $h^{\prime} F 2$ and $h_{t^{\prime 2}}^{\max }$ are about $2 \sim 4 \mathrm{~km}$, and maximum height is attained about 6 hours after lunar transit [15]. Therefore alike Martyn's, a conclusion might arise that a reverse lunar current flows both in $E$ and $F$ region. But for case (a), since $|B|$ is greater than $|A|$, as shown in Fig. 5, and $\varphi$ becomes about $70^{\circ}$, therefore upward maximum of $W$ is attained about 7 hours after lunar transit. Hence no contradiction will take place with $F 2$ variation, when the $E$ region is the main seat of the current.

When tidal oscillation in the $E$ region is $l_{L}$ times as great as that at the ground, amplitude of $W$ at $\theta=60^{\circ}$ is $0.527 l_{L} \mathrm{~cm} / \mathrm{sec}$. For case (b) a similar result is obtained.

As the observations of lunar $E$ variations are very few and detailed characteristics are not yet clear, we have hesitated to discuss them.

We tentatively suggest that the main seat of the lunar current is the $E$ region. 


\section{Variation of Conductivity depending on the Main Magnetic Field and $\lambda$}

We shall consider the variation of conductivity depending on main magnetic field and $\lambda$. Here we take the value of $D_{i}$ which was suggested by Tyndall (1938) [18] using observed mobility of $\mathrm{N}_{2}{ }^{+}$and that of $D_{e}$ which was shown by Huxley (1949) [19]. Molecular weights of ion and neutral molecule are taken to be 26, temperature of the gas to be $300^{\circ} \mathrm{K}$. Therefore we take the values $\nu_{e}=8.4 \times 10^{-9} \cdot n, \nu_{i}=5.09 \times 10^{-10} \cdot n$. We represent the $E$ layer at the equator at noon by a Chapman distribution with center at the gas density $n=10^{13}$ and for local scale height of $10^{6} \mathrm{~cm}$.

According to examination in section $2, \sigma^{I I I}$ will vary to some extent depending on the magnitude of $H$, since $\sigma^{I}$ varies little with $H$ but $\sigma^{I I}$ varies considerably with $\boldsymbol{H}$. We calculated conductivity of this layer using equ. (6), by numerical integration for various values of $\lambda$ and $H . \quad S_{3}$ denotes the case $H=0.3$ and $S_{4}$ the case $H=0.4$ (e.m.u.)

On the other hand we calculate conductivity $A$ of this layer using $\sigma^{I}$ and Formula by Appleton [20] for the case $\lambda=0$, and get the following values $A=A_{i}+A_{e}$ where $A_{i}=n_{e 0} \cdot 1.55 \times 10^{-14} H^{-\frac{1}{2}}, A_{e}=n_{e 0} \cdot 3.1 \times 10^{-16} H^{-2}$ with $n_{e 0}$ the maximum electron density of this layer.

When $\lambda \neq 0$, we assumed that $A=A_{e}+(2 \lambda+1) A_{i}$. The ratio $S / A$ with $\lambda$ is

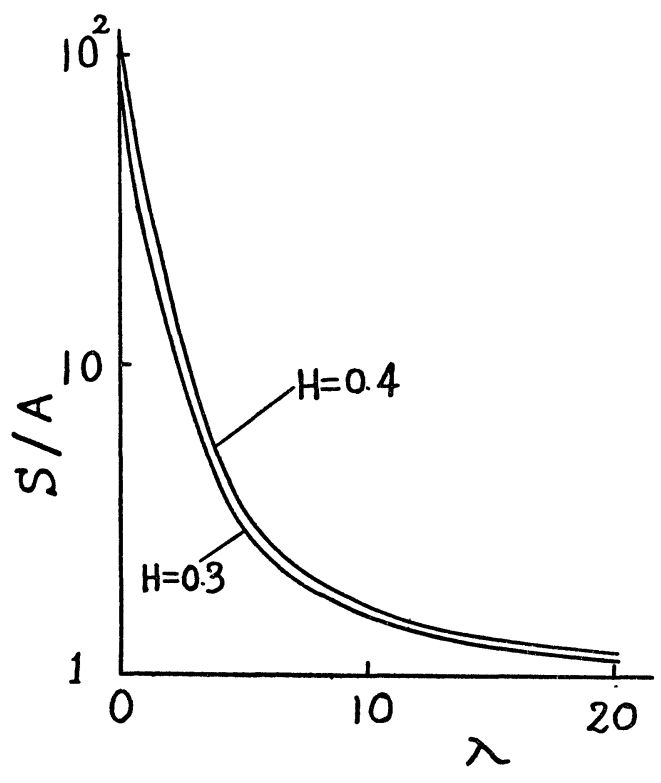

Fig. $7 S / A$ with the ratio of negative ions to electrons for various values of $H$.
Table 1

\begin{tabular}{c|c|c|c}
\hline$\lambda$ & 0 & 10 & 20 \\
\hline$S_{3} / S_{4}$ & 1.4 & 1.27 & 1.19
\end{tabular}

illustrated in Fig. 7 for $H=0.3$ and 0.4 . The ratio $S_{3} / S_{4}$ for various values of $\lambda$ is shown in Table 1.

The ratio $S / A$ may be a little smaller than curves in Fig. 7, because magnetic and electric fields are not completely uniform. When $\lambda \sim 10$, it can be shown that effective conductivity of the $E$ layer in middle latitudes is approximately equal to $A$. And moreover, the contribution of the $F 1$ layer to the Sq current will be present. Thus to explain observed data with the present theory, $\lambda$ should

be much smaller than 10 . According to Table $1, S$ decreases with increase of $H$, we imagine that difference of the ranges at Huancayo and at India might be due to this relation.

\section{Concluding Remarks}

In succession to two preliminary work I, II we further examined a theory that enhanced diurnal magnetic variations near the magnetic equator is an effect of high 
conductivity in $\mathrm{E}-\mathrm{W}$ direction due to the polarization field near the $E$ layer produced by Hall Current. The above calculation is made for the case that the axes of rotation and magnetism coincide. In the actual case that the two axes do not coincide and local anomalies are present, MS line will be along the magnetic equator (line of dip zero). If the concentration of the negative ions is effectively zero, the effective conductivity of the $E$ layer in middle latitudes is shown to be some 5 times that obtained by Appleton's formula. It is tentatively suggested that the main seat of the lunar current is the $E$ region, considering the vertical drift of the $F 2$ layer, produced by the polarization field due to the Hall Current. According to the present theory conductivity of the $E$ layer decreases with increase of the main magnetic field at the magnetic equator and observed distribution of ranges of diurnal variations reported by Egedal, seems to support this relation.

Moreover this theory is effective only when $\lambda$ is much smaller than 10 . Recent observed results seems to be favourable to the present theory. Further observations near the equators are very desirable.

\section{Acknowledgements}

The author wishes to express his thanks to Prof. M. Hasegawa for his continued interest and advice, and to Prof. T. Nagata for his valuable discussions during the progress of this work.

Many thanks are due to Dr. K. Maeda for his criticisms and helpful advice in this work.

\section{References}

[1] S. Hayami and H. Higashinaka, J. Shanghai Institute (in Japanese), Sec. I, 3, 129 (1934).

[2] M. Hasegawa and M. Ota, 'Transactions of Oslo meeting, 1948,' 426 (1950).

[3] M. Hasegawa, 'Transactions of Brussel meeting, 1951' (in the Press).

[4] A.T. Price, J. Geophys. Res., 56, No. 2, 259 (1951).

[5] J. Egedal, 'Transactions of Brussel meeting, 1951, (in the Press).

[6] M. Hirono, J. Geomag. Geoel. Kyoto, 2, 1 (1950).

[7] M. Hirono, J. Geomag. Geoel. Kyoto, 2, 113 (1950).

[8] D.R. Bates and H.S.W. Massey, Proc. Roy. Soc. Lon. A, 187, 261 (1946).

[9] T.G. Cowling, Proc. Roy. Soc. Lon. A, 183, 453 (1945).

[10] V.C.A. Ferraro, Terr. Mag. Washington D.C., 50, 215 (1945).

[11] K. Maeda, Read at the meeting of Ionosphere Research Committee, Feb. 29. 1952.

[12] S. Chapman and J. Bartels, 'Geomagnetism II,' 768 (1940).

[13] E.W. Hobson 'The Theory of Spherical and Ellipsoidal Harmonics' 89, Cambridge (1931).

[14] S. Chapman, Phil. Tran. Roy. Soc. Lon., 218, 1 (1919).

[15] D.F. Martyn, Proc. Roy. Soc. Lon., A, 194, 445 (1948).

[16] T.G. Cowling and R. Borger, Nature, Lon. 162, 143 (1948).

[17] D.R. Bates and H.S.W. Massey, J. Atmos. and Terr. Phys., 2, 1 (1951).

[18] A.M. Tyndall, Positive Ions, Camb. (1938).

[19] L.G.H. Huxley, A.A. Zaazou, Proc. Roy. Soc. Lon., 199, 402 (1949).

[20] E.V. Appleton, Proc, Roỵ. Soc. Lon. 162, 451 (1937).

(Read Apr. 28, 1952) 